\title{
SYNTHESIS AND ANTIMICROBIAL ACTIVITY OF NOVEL 1,3-OXAZOLIDINE NUCLEOSIDE ANALOGUES
}

\author{
S.N. Sriharsha and Sheena Shashikanth ${ }^{*}$ \\ Department of Studies in Chemistry, University of Mysore, Manasagangotri, Mysore -570 006, India \\ e-mail : skanth1@ rediffmail.com
}

\begin{abstract}
The synthesis of novel 1,3-oxazolidine pyrimidine nucleoside analogues are described. These analogues are all derived from the key stereochemically defined intermediate N-tert-butoxy-carbonyl-2hydroxymethyl-1,3-oxazolidine-4-ol which was accessible in $57 \%$ yield starting from L-serine methylester hydrochloride. The heterocyclic bases eg; uracil, thymine etc are efficiently introduced onto the 1,3-oxazolidine by the Vorbruggen procedures. The antimicrobial activity of novel 1,3-oxazolidine nucleoside analogues are highlighted. The compounds $\mathbf{7 d}$ and $7 \mathrm{e}$ showed significant activity against bacteria and fungus.

Key Words: 1,3-oxazolidine nucleosides, Antimicrobial activity.
\end{abstract}

\section{Introduction}

The potent activity displayed by $3^{\prime}$ - azido-3'-deoxythymidine (AZT) against human immunodifficiency virus ${ }^{1}$ (HIV) provides impetus for the development for the novel nucleoside analogues. ${ }^{2}$ Unfortunately, those compounds with the natural stereochemistry possess undesirable pharmacological properties and are susceptible to the development of resistant strains of HIV. In an attempt to overcome some of these detrimental side effects, the carbohydrate moiety of 2', 3'-dideoxy nucleoside analogues has been replaced by other five membered rings. ${ }^{3}$ It has been demonstrated that hetero substitution of these rings has a profound effect on the biological activity of the resultant nucleoside analogues as displayed by (-)-2'deoxy-3'-thiacytidine (3 TC) ${ }^{4}$ Antiviral nucleoside analogs containing more than one heteroatom in the sugar ring have received much attention because of their potent anti-HIV and anti-HBV activities. At present, $\beta$-L-(-)-2'-deoxy-3'-thiacytidine (Lamivudine) is clinically used for treatment of AIDS and AIDScomplex. Interestingly, this compound showed much lower cytotoxicity than its antipode the Denantiomer although both are almost equipotent against the replication of HIV-1 and -2 in vitro.

Oxazolidinones are the new class of antimicrobial agents with activity against broad spectrum of gram positive pathogens including staphalococci, streptococci and enterococci. ${ }^{5-7}$ The oxazolidinone structure is relatively simple and allows for diverse synthetic modification. Because of this and because of the importance of oxazolidinones as antimicrobial agents, we have efficiently introduced the heterocyclic bases eg; uracil, thymine etc onto the 1,3-oxazolidine by the Vorbruggen procedures ${ }^{8}$ and reported the antimicrobial activity of these compounds.

Our synthetic strategy was based on the preparation of N-tert-butoxycarbonyl-1,3-oxazolidine-4-one (2) followed by the reduction and acylation to give 4-acyloxy-1,3-oxazolidine. Thus compound 2 has been prepared by condensing di-tert-butyl-di-carbonate with L-serine methyl ester hydrochloride(1) in the presence of dimethylaminopyridine (DMAP) and triethylamine. ${ }^{9}$ The lactam and the ester functional groups present has been reduced by using $\mathrm{Li}(\mathrm{et}){ }_{3} \mathrm{BH}$ at $-78^{\circ} \mathrm{C}$ to get the desired N-tert-butoxycarbonyl-2hydroxymethyl-1,3-oxazolidine-4-ol(3)in quantitative yield. ${ }^{10-12}$ Protection of primary alcoholic group using trityl chloride has been carried out in the presence of pyridine to give trityl derivative (4). This was subjected to acetylation in the presence of acetic anhydride, dimethylaminopyridine and triethylamine to give N-tert-butoxycarbonyl-2-hydroxymethyl- 4-acyloxy-1,3-Oxazolidine (5) ${ }^{13}$ (Scheme-1) 


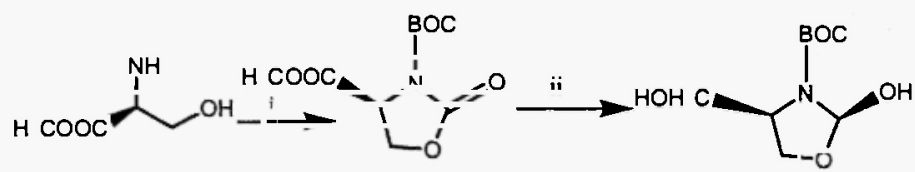

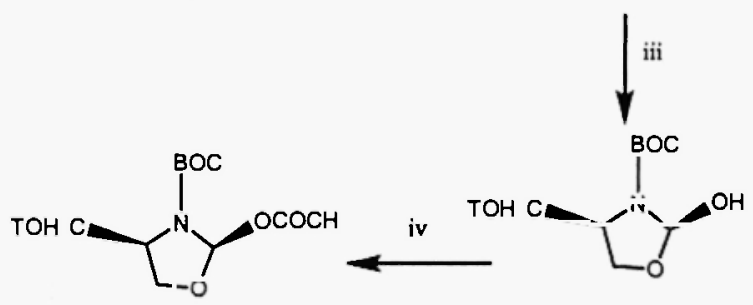

ents and Conditions:

i) (BOC) O, DMAP, (Et) N, RT ii) Li(et) BH, THF, - C iii) Trityl Chloride, Pyridine, Reflux iv) (CH CO) O, DMAP, (Et) N, RT.

\section{Scheme-1}

The Compound $\mathbf{5}$ is suitable for coupling with silylated pyrimidine bases under refluxing conditions in $\mathrm{CH}_{2} \mathrm{CN}$ and in the presence Stannic Chloride as lewis acid (Scheme-2). This gave the desired nucleoside analogues as an anomeric mixture in moderate yields. ${ }^{14-15}$. The ${ }^{1} \mathrm{H}$ NMR spectra of the nucleoside analogues (7a-f) showed multiplet due to the aromatic protons in the region $6.55-8.10$. The signals due to the $\mathrm{NH}$ protons were appeared in the region at 10.0 and the $\mathrm{C}^{\mathrm{l}}-\mathrm{H}$ proton of the oxazolidine moiety caused a singlet in the region 5.05 indicating the attachment of the oxazolidine moiety and the formation of configuration. While singlet due to the $\mathrm{OH}$ protons in these compounds was noticed at 2.0. The N-tertbutoxycarbonyl (BOC) and trityl ( $\mathrm{T}$ ) groups are deprotected by stirring it with trifluoroacetic acid at room temperature. The structures of the synthesized compounds have been characterized by the spectroscopic data and elemental analysis. The results are summarized in Table-1.

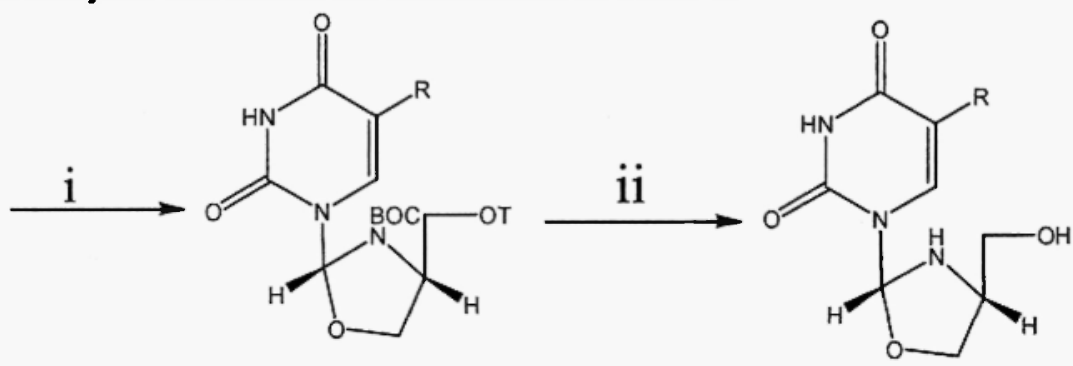

i) Silylated pyrimidine base, TMSOTf or $\mathrm{SnCl}, \mathrm{CH} \mathrm{CN}, \mathrm{O}$ C ii ) TriflouroAcetic acid, RT.

Base = a) $\mathrm{R}=\mathrm{H}$ Uracil, b) $\mathrm{R}=\mathrm{CH}$ Thymine, c) $\mathrm{R}=\mathrm{SH}$, -ThioUracil, d) $\mathrm{R}=\mathrm{F}$-Flourouracil, e) $\mathrm{R}=\mathrm{Br} \quad$-BromoUracil, f) $\mathrm{R}=\mathrm{Cl} \quad$-ChloroUracil etc

\section{Scheme-2}

\section{Antimicrobial Activity}

All the synthesized nucleosides were screened for their antimicrobial activity against bacteria E.coli (gram negative), S.aureus (gram positive) and fungi viz A. niger, C.albicans and A.flavus, at the conc of $100 \mu \mathrm{g} / \mathrm{ml}$, using Norfloxacin and Griseofulvin respectively as reference compounds. These investigations have been performed by disc diffusion method by Verma et al. ${ }^{16}$ The test compounds were dissolved in dimethyl formamide and different aliquots were placed in each cup. Incubation was carried out at $37^{\circ} \mathrm{C}$ for $24 \mathrm{hr}$. The results are summarized in table- 2 . 
Table-1: Analytial and spectral data

\begin{tabular}{|c|c|c|c|c|c|}
\hline $\begin{array}{l}\text { Compd. } \\
\text { No }\end{array}$ & $\begin{array}{l}\text { Yield } \\
(\%)\end{array}$ & $\begin{array}{l}\text { m.p in } \\
{ }^{\circ} \mathrm{C}\end{array}$ & $\begin{array}{c}\mathrm{C}, \mathrm{H}, \mathrm{N} \text { Analysis } \\
\text { Anal Caled for }\end{array}$ & IR (nujol) $\mathrm{cm}^{-1}$ & ${ }^{\top} \mathrm{H}$ NMR $\left(\mathrm{CDCl}_{3}\right)$ \\
\hline 2 & 60 & 68 & $\begin{array}{l}\mathrm{C}_{10} \mathrm{H}_{15} \mathrm{NO}_{6} \\
\mathrm{C}, 48.98 ; \mathrm{H}, 6.17 ; \mathrm{N}, 5.71 \\
\text { Found; } \mathrm{C} 48.95 ; \mathrm{H}, 6.15 ; \mathrm{N}, 5.70\end{array}$ & $\begin{array}{l}1710-1720 \mathrm{~cm}^{-1}(\mathrm{C}=\mathrm{O} \text { of } \mathrm{BOC} \text {-ester } \\
\text { group) } \\
1820 \mathrm{~cm}^{-1}(\mathrm{C}=\mathrm{O} \text { group }), \\
1690 \mathrm{~cm}^{-1}(\mathrm{C}=\mathrm{O} \text { group }) .\end{array}$ & $\begin{array}{l}4.99 \text { (1H,dd), } \\
3.84(3 \mathrm{H}, \mathrm{s}), \\
3.64(1 \mathrm{H}, \mathrm{dd}), 3.32 \text { (1H,dd), } \\
1.51,(9 \mathrm{H} \mathrm{s}, \text { Boc ester })\end{array}$ \\
\hline 3 & 57 & 79 & $\begin{array}{l}\mathrm{C}_{9} \mathrm{H}_{19} \mathrm{NO}_{5 . .} \\
\mathrm{C}, 49.31 ; \mathrm{H}, 7.76 ; \mathrm{N}, 6.39 \\
\text { Found; } \mathrm{C}, 49.29 ; \mathrm{H}, 7.74 ; \mathrm{N} ; 6.36 .\end{array}$ & $\begin{array}{l}1710-1720 \mathrm{~cm}^{-1}(\mathrm{C}=\mathrm{O} \text { of } \mathrm{BOC} \text {-ester } \\
\text { group), } 3200-3220 \mathrm{~cm}^{-1}(\mathrm{OH})\end{array}$ & $\begin{array}{l}4.99(1 \mathrm{H}, \mathrm{dd}), \\
3.64(2 \mathrm{H}, \mathrm{dd}), 1.51,(9 \mathrm{H} \mathrm{s}, \mathrm{C}=0) 2.78 \\
(\mathrm{~d}, 2 \mathrm{H}) 6.68(1 \mathrm{H}, \mathrm{s}, \mathrm{CH}) \\
2(2 \mathrm{H}, \mathrm{s}, \mathrm{OH})\end{array}$ \\
\hline 4 & 65 & 112 & $\begin{array}{l}\mathrm{C}_{28} \mathrm{H}_{31} \mathrm{NO}_{5} \\
\mathrm{C}, 72.86 ; \mathrm{H}, 6.77 ; \mathrm{N}, 3.03 \\
\text { Found; } 72.85 ; \mathrm{H}, 6.75 ; \mathrm{N}, 3.04 .\end{array}$ & $\begin{array}{l}1710-1720 \mathrm{~cm}^{-1}(\mathrm{C}=\mathrm{O} \text { of BOC-ester } \\
\text { group), } 3200-3220 \mathrm{~cm}^{-1}(\mathrm{OH}) \\
750 \mathrm{~cm}^{-1}(\mathrm{ArH}) .\end{array}$ & $\begin{array}{l}4.99 \text { (lH,dd), } \\
3.64(1 \mathrm{H}, \mathrm{dd}), \\
1.5 \mathrm{l},(9 \mathrm{H} \mathrm{s}, \text { Boc ester }), 2.78(\mathrm{~d}, 2 \mathrm{H}) \\
6.68(\mathrm{H}, \mathrm{s}, \mathrm{CH}) \\
2(2 \mathrm{H}, \mathrm{s}, \mathrm{OH}) \\
7.19(15 \mathrm{H}, \mathrm{s}, \text { ArH })\end{array}$ \\
\hline 5 & 53 & 130 & $\begin{array}{l}\mathrm{C}_{30} \mathrm{H}_{33} \mathrm{NO}_{6} \\
\mathrm{C}, 72.55: \mathrm{H}, 6.61 ; \mathrm{N}, 2.78, \text { Found; } 72.56 \\
\mathrm{H}, 6.62 ; \mathrm{N}, 2.79 .\end{array}$ & $\begin{array}{l}1710-1720 \mathrm{~cm}^{-1}(\mathrm{C}=\mathrm{O} \text { of BOC-este group), } \\
1690(\mathrm{C}=\mathrm{O} \text { of actcory } \mathrm{gp}) 750 \mathrm{~cm}^{-1}(\mathrm{ArH})\end{array}$ & $\begin{array}{l}4.99(1 \mathrm{H}, \mathrm{dd}), \\
3.64(\mathrm{lH}, \mathrm{dd}), 2.01(3 \mathrm{H}, \mathrm{s}) 2.78(\mathrm{~d}, 2 \mathrm{H}) \\
1.51,(9 \mathrm{H}, \mathrm{s}, \mathrm{Boc} \text { ester } \\
6.68(\mathrm{H}, \mathrm{s}, \mathrm{CH}) \\
2(2 \mathrm{H}, \mathrm{s}, \mathrm{OH}) \\
7.1(15 \mathrm{H}, \mathrm{s}, \mathrm{ArH})\end{array}$ \\
\hline $7 a$ & 50 & - & $\begin{array}{l}\mathrm{C}_{\mathrm{B}} \mathrm{H}_{\mathrm{II}} \mathrm{N}, \mathrm{O}_{4} \\
\mathrm{C}, 45.07 ; \mathrm{H}, \mathrm{S} .16 ; \mathrm{N}, 19.71, \\
\text { Found; } \mathrm{C}, 45.03 ; \mathrm{H}, 5.12 ; \mathrm{N}, 19.70 .\end{array}$ & $\begin{array}{l}1890 \mathrm{~cm}^{-1}(\mathrm{C}=0), \\
1690 \mathrm{~cm}^{-1}(\mathrm{C}=0), \\
2950 \mathrm{~cm}^{-1}(\mathrm{NH}) \\
3557 \mathrm{~cm}^{-1}(\mathrm{OH})\end{array}$ & $\begin{array}{l}10.0\left(1 \mathrm{H}_{\mathrm{s}} \mathrm{s}, \mathrm{NH}\right), 5.05(\mathrm{HH}, \mathrm{CH}) \\
4.05\left(2 \mathrm{H}, \mathrm{t}, \mathrm{H}_{2}\right) \\
3.84(2 \mathrm{H}, \mathrm{t}, \mathrm{H} 2) \\
2.0(1 \mathrm{H}, \mathrm{s}, \mathrm{OH}) \\
2.3(1 \mathrm{H}, \mathrm{s}, \mathrm{NH}) \\
5.76(1 \mathrm{H}, \mathrm{d}, \mathrm{CH}), 7.79(1 \mathrm{H}, \mathrm{d}, \mathrm{CH})\end{array}$ \\
\hline $7 b$ & 45 & - & $\begin{array}{l}\mathrm{C}_{8} \mathrm{H}_{41} \mathrm{~N}_{3} \mathrm{O}_{4}, \\
\mathrm{C}, 42.02 ; \mathrm{H}, 4.28 ; \\
\mathrm{N}, 16.34, \mathrm{~S}, 12.45 \\
\text { Found; } \mathrm{C}, 42.05 ; \mathrm{H}, 4.26 ; \mathrm{N}, 16.30, \mathrm{~S} \text {, } \\
12.40 \text {. }\end{array}$ & $\begin{array}{l}1890 \mathrm{~cm}^{-1}(\mathrm{C}=\mathrm{O}), \\
1690 \mathrm{~cm}^{-1}(\mathrm{C}=\mathrm{O}), \\
2950 \mathrm{~cm}^{-1}(\mathrm{NH}) \\
3557 \mathrm{~cm}^{-1}(\mathrm{OH})\end{array}$ & $\begin{array}{l}10.0(1 \mathrm{H}, \mathrm{s}, \mathrm{NH}) \\
5.05(1 \mathrm{H}, \mathrm{t}, \mathrm{CH}) \\
4.05\left(2 \mathrm{H}, \mathrm{t}, \mathrm{H}_{2}\right) \\
3.84\left(\mathrm{HH}, \mathrm{t}, \mathrm{H}_{2}\right) \\
2.0(1 \mathrm{H}, \mathrm{s}, \mathrm{OH}) \\
2.3(1 \mathrm{H}, \mathrm{s}, \mathrm{NH}) \\
1.93\left(3 \mathrm{H}, \mathrm{d}, \mathrm{CH}_{3}\right) \\
7.57(1 \mathrm{H}, \mathrm{s}, \mathrm{CH})\end{array}$ \\
\hline $7 c$ & 52 & - & $\begin{array}{l}\mathrm{C}_{8} \mathrm{H}_{11} \mathrm{~N}_{3} \mathrm{O}_{4} \mathrm{~S}, \\
\mathrm{C}, 42.02 ; \mathrm{H}, 4.28 ; 16.34, \mathrm{~S}, 12.45, \\
\text { Found; } \mathrm{C}, 42.05 ; \mathrm{H}, 4.26 ; \mathrm{N}, 16.30, \mathrm{~S} \text {, } \\
12.40\end{array}$ & $\begin{array}{l}1890 \mathrm{~cm}^{-1}(\mathrm{C}=0), \\
1690 \mathrm{~cm}^{-1}(\mathrm{C}=\mathrm{O}), 2950 \mathrm{~cm}^{-1}(\mathrm{NH}), \\
3557 \mathrm{~cm}^{-1}(\mathrm{OH}), \\
2600 \mathrm{~cm}^{-1}(\mathrm{SH})\end{array}$ & $\begin{array}{l}10.0(1 \mathrm{H}, \mathrm{s}, \mathrm{NH}) \\
1.5(1 \mathrm{H}, \mathrm{dSH}) \\
5.05(1 \mathrm{H}, \mathrm{t}, \mathrm{CH}), 4.05\left(2 \mathrm{H}, \mathrm{t}, \mathrm{H}_{2}\right) \\
3.90\left(1 \mathrm{H}, \mathrm{t}, \mathrm{H}_{2}\right) \\
3.84\left(2 \mathrm{H}, \mathrm{d}, \mathrm{H}_{2}\right) \\
2.0(1 \mathrm{H}, \mathrm{s}, \mathrm{OH}) \\
2.3(1 \mathrm{H}, \mathrm{s}, \mathrm{NH}) \\
4.5(1 \mathrm{H}, \mathrm{d}, \mathrm{lH}) \\
7.3(1 \mathrm{H}, \mathrm{d}, \mathrm{CH})\end{array}$ \\
\hline $7 d$ & 48 & $\cdots$ & $\begin{array}{l}\mathrm{C}_{8} \mathrm{H}_{10} \mathrm{FN}_{3} \mathrm{O}_{4} \\
\mathrm{C}, 40.16 ; \mathrm{H}, 4.18 ; \mathrm{N}, 17.57, \mathrm{~F}, 11.29 \\
\text { Found; } \mathrm{C}, 40.17 ; \mathrm{H}, 4.17 \\
\mathrm{~N}, 17.50, \mathrm{~F}, 11.27\end{array}$ & $\begin{array}{l}1890 \mathrm{~cm}^{-1}(\mathrm{C}=0) \\
1690 \mathrm{~cm}^{-1}(\mathrm{C}=\mathrm{O}) \\
2950 \mathrm{~cm}^{-1}(\mathrm{NH}) \\
3557 \mathrm{~cm}^{-1}(\mathrm{OH})\end{array}$ & $\begin{array}{l}10.0(\mathrm{lH}, \mathrm{s}, \mathrm{NH}) \\
5.05(1 \mathrm{H}, \mathrm{t}, \mathrm{CH}) \\
4.06\left(2 \mathrm{H}, \mathrm{t}, \mathrm{H}_{2}\right) \\
3.90\left(1 \mathrm{H}, \mathrm{t}, \mathrm{H}_{2}\right) \\
3.84\left(2 \mathrm{H}, \mathrm{d}, \mathrm{H}_{2}\right) \\
2.0(1 \mathrm{H}, \mathrm{s}, \mathrm{OH}) \\
2.3(1 \mathrm{H}, \mathrm{s}, \mathrm{NH}) \\
7.39(\mathrm{HH}, \mathrm{s}, \mathrm{CH})\end{array}$ \\
\hline $7 e$ & 51 & - & $\begin{array}{l}\mathrm{C}_{8} \mathrm{H}_{10} \mathrm{BrN}_{3} \mathrm{O}_{4} \\
\mathrm{C}, 32.87 ; \mathrm{H}, 3.42 ; \\
\mathrm{N}, 14.38 ; \mathrm{Br} ; 27.39 \\
\text { Found; } \mathrm{C}, 32.85 ; \mathrm{H}, 3.32 \\
\mathrm{~N}, 14.30 ; \mathrm{Br} ; 27.38\end{array}$ & $\begin{array}{l}1890 \mathrm{~cm}^{-1}(\mathrm{C}=\mathrm{O}), \\
1690 \mathrm{~cm}^{-1}(\mathrm{C}=\mathrm{O}), \\
2950 \mathrm{~cm}^{-1}(\mathrm{NH}) \\
3557 \mathrm{~cm}^{-1}(\mathrm{OH})\end{array}$ & $\begin{array}{l}10.0(1 \mathrm{H}, \mathrm{s}, \mathrm{NH}) \\
5.05(\mathrm{lH}, \mathrm{t}, \mathrm{CH}) \\
4.06(2 \mathrm{H}, \mathrm{t, \textrm {H }}) \\
3.90\left(1 \mathrm{H}, \mathrm{t}, \mathrm{H}_{2}\right) \\
2.0(1 \mathrm{H}, \mathrm{s}, \mathrm{OH}) \\
2.3(1 \mathrm{H}, \mathrm{s}, \mathrm{NH}) \\
8.24(1 \mathrm{H}, \mathrm{s}, \mathrm{CH})\end{array}$ \\
\hline $7 \mathrm{f}$ & 62 & - & $\begin{array}{l}\mathrm{C}_{8} \mathrm{H}_{10} \mathrm{ClN}_{3} \mathrm{O}_{4} \\
\mathrm{C}, 38.70 ; \mathrm{H}, 4.03 ; \mathrm{N}, 16.93 ; \mathrm{Cl}, 14.51 \\
\text { Found } \mathrm{C}, 38.73 ; \mathrm{H}, 4.05 ; \mathrm{N}, 6.90 ; \mathrm{Cl}, 14.5\end{array}$ & $\begin{array}{l}1890 \mathrm{~cm}^{-1}(\mathrm{C}=\mathrm{O}) \\
1690 \mathrm{~cm}^{-1}(\mathrm{C}=\mathrm{O}) \\
2950 \mathrm{~cm}^{-1}(\mathrm{NH}) \\
3557 \mathrm{~cm}^{-1}(\mathrm{OH})\end{array}$ & $\begin{array}{l}10.0(\mathrm{lH}, \mathrm{s}, \mathrm{NH}) \\
5.05(1 \mathrm{H}, \mathrm{t}, \mathrm{CH}) \\
4.06\left(2 \mathrm{H}, \mathrm{t}, \mathrm{H}_{2}\right) \\
3.84\left(1 \mathrm{H}, \mathrm{t}, \mathrm{H}_{2}\right) \\
2.0(1 \mathrm{H}, \mathrm{s}, \mathrm{OH}) \\
2.3(1 \mathrm{H}, \mathrm{s}, \mathrm{NH}) \\
8.24(1 \mathrm{H}, \mathrm{s}, \mathrm{CH})\end{array}$ \\
\hline
\end{tabular}


Table-2: Effect of compounds 7a-f on bacterial and fungal activity ( Dilution: $100 \mu \mathrm{g} / \mathrm{ml}$.)

\begin{tabular}{|c|c|c|c|c|c|}
\hline \multirow{2}{*}{ Compounds } & \multicolumn{3}{|c|}{ Antibacterial activity } & \multicolumn{3}{c|}{ Antifungal activity } \\
\cline { 2 - 6 } & \multicolumn{2}{|c|}{ Zone of Inhibition(mm) } & \multicolumn{2}{c|}{ Zone of Inhibition (mm) } \\
\hline & E.Coli & S.Aureus & A.niger & C.albicans & A.flavus \\
\hline 7a & 23 & 24 & 24 & 22 & 23 \\
\hline 7b & 25 & 24 & 25 & 24 & 26 \\
\hline 7c & 25 & 26 & 26 & 27 & 27 \\
\hline 7d & 34 & 30 & 31 & 30 & 31 \\
\hline 7e & 32 & 30 & 30 & 30 & 31 \\
\hline 7f & 30 & 29 & 28 & 30 & 29 \\
\hline Norfloxacin & 35 & 32 & ---- & - & - \\
\hline Griseofulvin & -- & -- & 32 & 32 & 32 \\
\hline
\end{tabular}

\section{Experimental}

The melting points were determined in open capillary and are uncorrected. The IR spectra were recorded on Shimatzu FT-IR spectrophotometer in nujol. ${ }^{\mathrm{l}} \mathrm{H}$ NMR spectra was recorded on Hitatchi R-600 spectrometer with TMS as internal standard.

\section{N-tert-butoxycarbonyl-4-(methoxy carbonyl)-1,3-oxazolidin-2-one (2)}

Di-tert-butyl-di-carbonate ( 3 equiv) was dissolved in $4 \mathrm{ml}$ of $\mathrm{MeCN}$ (or toluene) and placed in an ice bath and 0.5 equiv of DMAP and 3 equiv of triethylamine were added. After 5 minutes Serine methyl ester hydrochloride $(0.083 \mathrm{~g}, 0.5 \mathrm{mmol})$ was added in portions for during 2 mins and the reaction was allowed to run for 1 hour more. At the end of the reaction chloroform was added and the solution was washed with $5 \% \mathrm{HCl}(2 \times 20 \mathrm{ml})$, dried with $\mathrm{MgSO}_{4}$ and evaporated to give the compound 2 as a white solid in good yield.

\section{N-tert-butoxycarbonyl-4-(hydroxyl methyl)-1,3-oxazolidin-2-ol (3)}

Compound 2 was dissolved in THF $(20 \mathrm{ml})$, cooled to $-78^{\circ} \mathrm{C}$ was added 3 eq of $1 \mathrm{M}$ solution of $\mathrm{Li}(\mathrm{et}){ }_{3} \mathrm{BH}$. The reaction requires 10 to 60 mins for completion. Excess of reagent was destroyed by the addition of saturated solution of $\mathrm{NH}_{4} \mathrm{Cl}$ at $-78^{\circ} \mathrm{C}$ and the reaction mixture was extracted with dichloromethane $(3 \times 15 \mathrm{ml})$ and dried over anhydrous $\mathrm{MgSO}_{4}$ and evaporated to dryness followed by the purification by flash chromatography (hexane-ethyl acetate 7:2) afford the compound 3 as light yellow oil.

\section{N-tert-butoxycarbonyl-4-(trityloxy methyl)-1,3-oxazolidine-2-ol (4)}

Compound $3(0.8979 \mathrm{~g}, 4 \mathrm{mmol})$ and trityl chloride (triphenylmethylchloride) $(1.4 \mathrm{~g}, 5 \mathrm{mmol})$ were dissolved in $20 \mathrm{ml}$ of pyridine and the mixture was heated at $100^{\circ} \mathrm{C}$ (steam bath) with swirling for 30 mins. The reaction mixture was cooled at room temperature and then poured in to $100 \mathrm{ml}$ of ice water. The slurry was stirred vigorously during quenching. The solid was filtered and washed thoroughly with water until it is free from pyridine. Dry the solid separately. Finally recrystallization from acetone-toluene to give a white solid of O-trityl derivative.

4 N-tert-butoxycarbonyl-2-hydroxymethyl- 4-acyloxy-1,3-oxazolidine (5).

A solution of trityl derivative 4 in $\mathrm{CH}_{2} \mathrm{Cl}_{2}$ was treated with acetic anhydride (2.6gm,26.3 mmol), Triethylamine $(2.6 \mathrm{gm}, 26.3 \mathrm{mmol})$ and a catalytic amount of 4-DMAP at room temperature for $3 \mathrm{hrs}$. The 
resultant mixture was washed with $5 \% \mathrm{HCl}(3 \times 15 \mathrm{ml})$ and extracted with $\mathrm{CH}_{2} \mathrm{Cl}_{2}(3 \times 20 \mathrm{ml})$ and evaporated to dryness and purified by silica gel column chromatography with $5 \%$ chloroform: Ethyl acetate $7: 2$ to get white solid.

\section{General procedure for the synthesis of 1,3-oxazolidine nucleoside analogues (7a-f)}

A mixture of pyrimidine base $(2.35 \mathrm{mmol})$ in Hexamethyldisilazane $(10 \mathrm{ml})$ and $\mathrm{CH}_{3} \mathrm{CN}$ was heated under reflux for $5 \mathrm{hrs}$. After removal of solvent by vacuum pump, a solution of compound 5 ( 0.786 $\mathrm{mmol}$ ) in $15 \mathrm{ml}$ of $\mathrm{CH}_{3} \mathrm{CN}$ was added to the reaction flask containing the silylated pyrimidine bases and then Stannic Chloride $(1.4 \mathrm{mmol})$ was added dropwise at room temperature. After 16 hrs the reaction was quenched with $1 \mathrm{ml}$ of saturated $\mathrm{NaHCO}_{3}$ and the resultant mixture was concentrated. The crude mixture was exctracted with $\mathrm{CH}_{2} \mathrm{Cl}_{2}(3 \times 20 \mathrm{ml})$, washed with aq. $\mathrm{NaHCO}_{3}$, dried over $\mathrm{MgSO}_{4}$, filterd and concentrated. The crude product was purified by silica gel column chromatography with ethyl acetate:hexane (7:2) to give nucleoside analogues (6a-f) as yellow oil. Finally BOC and trityl groups were deprotected by stirring it with triflouroacetic acid at room temperature afford $7 \mathbf{a}-\mathbf{f}$ in moderate yield.

\section{Conclusions}

In conclusion, we have presented short and efficient preparations of the 1,3-oxazolidine nucleoside analogues which as a consequence of their low toxicity should prove to be important antimicrobial agents. The novel 1,3-oxazolidine nucleoside analogues have been synthesized using Vorbruggen procedure by condensing silylated pyrimidine bases with the acylated 1,3-oxazolidine moiety in the presence of lewis acid to get the desired products in the moderate yield. The antimicrobial activity of these compounds have been studied and in that compounds $7 \mathbf{d}$ and $7 \mathrm{e}$ with fluoro and bromo substituents showed significant activity against E.coli, Saureus, $A$. niger, C.albicans and $A$.flavus among the six compounds screened.

\section{Acknowledgements}

SNS thank CSIR, Government of India, New Delhi for Senior Research Fellowship and acknowledge Dr.K.R.Prabhu, senior scientific officer, Dept of organic chemistry, IISC, Bangalore for recording IR and NMR spectra. Authors are thankful to Mr.Vijay Kumar, Asst.Prof., National College of Pharmacy, Shimoga, for carrying out the antimicrobial activity.

\section{References}

1. H. Mitsuya, J.K. Wienhold, P.A.Furman, H St-clair, S. Nusinoff Lehman, R.C. Gallo D.bolognesi and S. Broader, Proc. Natl. Acad. Sci., USA, 82, 7096 (1985).

2. H. Mitsuya and S. Border, Proc. Natl. Acad. Sci., USA, 83, 1911 (1986)

3. T.S. Linn, R.F. Schinazi and W.H. Prushoff, Biochem Pharmacol, 36, 2713 (1987)

4. M.L. Peterson and R.Vince, J. Med. Chem. 37, 2787 (1991)

5. S.J. Brickner, D.K. Hutchinson, M.R. Barbachyn, P.R. Manninen, D.A. Ulanowicz, Antimicrob. Agents Chemother. 39, 673 (1996)

6. B. Belleau, L. Brasilli, L. Chan, B. Zacheri, J. Cameron, Bio-org. Med. Chem Lett. 3, 1723 (1993) 
7. Nghe Nguyen-Ba, Willium L. Brown, Laval Chan, Nola Lee, Livio Brasilli, Dominique Lafleur and Boulous Zacharie, Chem. Commun. 1245 (1999)

8. H.Vorbruggen, K.Krolikiewicz and B. Bennua, Chem. Ber, 114, 1234 (1981)

9. Yochai Basel and Alfred Hassner, J. Org. Chem. 65, 6368 (2000)

10. S.K. Chaudhary, Hernandez, Tetrahedron Lett. 20, 95 (1979)

11. Mark L. Peterson and Robert Vince, J. Med. Chem. 34, 2787 (1991)

12. Herbert C. Brown, S.C. Kim and S. Krishnamurthy, J. Org. Chem. 45, 1 (1980)

13. Youhoon Chong, Hyunah Choo and Yongseok Choi and Raymond Schinazi Chaung Chu 45, 4888 (2002).

14. N. Nguyen-Ba, W. Brown, N. Lee and B. Zacharie, Synthesis 759 (1998)

15. M.Therien, G.Y.Gauthier and R.N. Young, Tetrahedron Lett 29, 6733 (1988)

16. R.S.Varma and W.L.Nobles, J. Pharma Sci. 61,112 (1972)

Received on November 15, 2005 